\title{
Mapping the Nanoscale Redshift of Optical Phonon Modes in a Strained Quantum Dot System
}

\author{
Chaitanya Gadre ${ }^{1}$, Xingxu Yan ${ }^{2}$, Toshihiro Aoki ${ }^{3}$, Sheng-Wei Lee ${ }^{2,4}$ and Xiaoqing Pan ${ }^{1,2,3}$ \\ 1. Department of Physics and Astronomy, University of California-Irvine, Irvine, CA, USA. \\ 2. Department of Materials Science and Engineering, University of California-Irvine, Irvine, CA, USA. \\ 3. Irvine Materials Research Institute, University of California-Irvine, Irvine, CA, USA. \\ 4. Institute of Materials Science and Engineering, National Central University, Taoyuan, Taiwan.
}

Thermoelectric materials have garnered a lot of interest in an era where energy is in short supply. Such materials allow for the direct conversion of heat to electricity, consequently providing a great alternative for power generation and other applications [1]. Recently, much effort has been invested in optimizing the figure-of-merit $(Z T)$ of thermoelectric materials producing novel nanostructures with low thermal and high electrical conductivity [2]. Strain and interface dynamics play a paramount role in the performance of a thermoelectric. In general, compressive strain can increase thermal conductivity while tensile strain decreases thermal conductivity, consequently enhancing energy conversion efficiency [3]. The $\mathrm{Si} / \mathrm{Ge}$ multi-layered quantum dot (QD) system is a promising nanostructured material for thermoelectric applications due to the lattice parameter discrepancy between silicon and germanium. The resulting tensile strain on the intermixed silicon inside the $\mathrm{Si} / \mathrm{Ge}$ QD decreases the thermal conductivity and increases thermoelectric performance. These properties have been studied and optimized allowing for increased phonon scattering and decreased thermal conductivity but haven't been explored at the atomic resolution.

For such nanoscale structures, it becomes necessary to properly probe and quantify the local effects of strain due to the intermixing between silicon and germanium to optimize thermal efficiency. Traditional optical spectroscopic methods have been employed to study large-scale characteristics but lack the necessary spatial resolution to quantify properties of a single QD, Electron energy loss spectroscopy (EELS) in a high energy resolution monochromated EELS system (HERMES) is aptly poised to investigate nanoscale features at a high spatial and energy resolution. This technique has already been used to investigate several materials [4] and has now been shown to be capable of probing local vibrational modes at atomic resolution [5]. Utilizing this state-of-the-art technique, local strain effects due to the $\mathrm{Si} / \mathrm{Ge}$ intermixing are demonstrated for the first time using vibrational spectroscopy at a high spatial resolution.

In order to study this effect precisely, high quality spectra were acquired by summing individual one second exposure spectra using Nion UltraSTEM 200 HERMES operating at $60 \mathrm{keV}$. After background subtraction and peak separation, information was obtained on each of the individual peaks listed in Fig. 2a. The emergence of the Ge-Ge vibrational peak is clearly visible with the beam placed inside the dot. A nanometer step line scan, shown in Fig. 2b, was obtained revealing the Si TO/LO mode redshift inside the QD and is a consequence of the Si-Ge intermixing. Elemental mapping shown in Fig. 1b illustrate the relative composition of the QD. Varying levels of Ge inside the QD will cause a proportional redshift of the Si transverse/longitudinal optical (TO/LO) degenerate phonon mode. Fig. 1a shows an atomic resolution high angle annular dark field (HAADF) image of the $\mathrm{Si} / \mathrm{Ge} \mathrm{QD}$ containing an interface as sharp as 1-2 nm which is also illustrated in the abrupt energy change in the $\mathrm{Si} \mathrm{TO} / \mathrm{LO}$ mode. This work demonstrates for the first time that, with the ability to probe highly localized properties, it is possible to investigate strain as well as interface dynamics as a function of QD composition in the nanometer scale [6]. 
References:

[1] K Biswas et al., Nature 489.7416 (2012), p. 414.

[2] H Chang et al., Nanoscale 6.7 (2014), p. 3593.

[3] X Li et al., Physical Review B 81.24 (2010), p. 245318.

[4] OL Krivanek et al., Nature 514.7527 (2014), p. 209.

[5] FS Hage et al., Physical Review Letters 122 (2019), p. 016103.

[6] This work was supported by the Department of Energy (DOE) under Grant DE-SC0014430.

The authors acknowledge the support of the University of California Irvine Materials Research Institute for the use of TEM facilities.
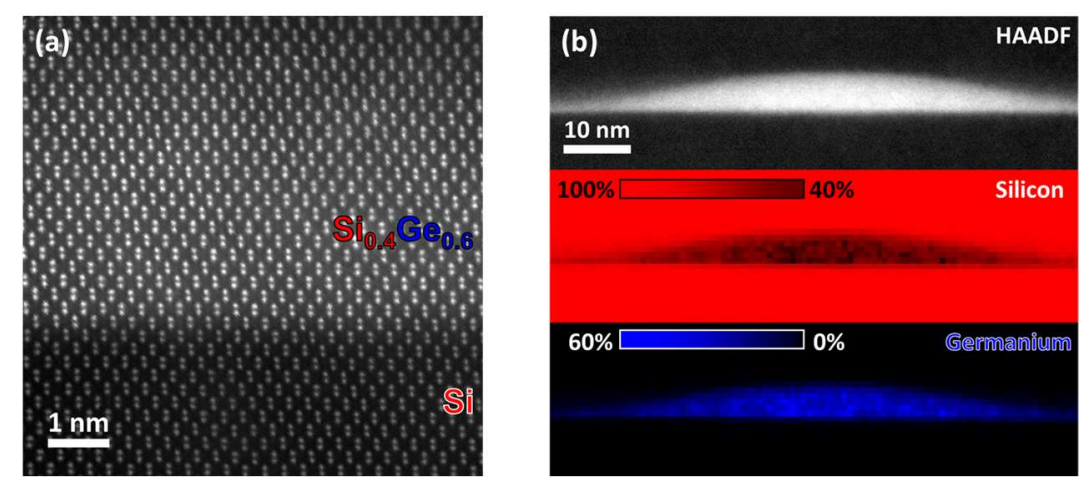

Figure 1. Imaging and elemental mapping of the Si/Ge QD. (a) Atomic resolution HAADF image of $\mathrm{Si} / \mathrm{Ge}$ interface. The upper part (bright) is the germanium and the lower part (darker) corresponds to the interlayer deposited silicon. Scale bar: $1 \mathrm{~nm}$. (b) Top: HAADF image of a single Si/Ge QD; middle: Si $K$ edge map; bottom: Ge $L_{2,3}$ edge map. The red area is pure silicon and the blue area is predominantly germanium with some silicon intermixing. Scale bar: $10 \mathrm{~nm}$. The scales on the top of Si and Ge elemental maps show the composition in atomic percentage.
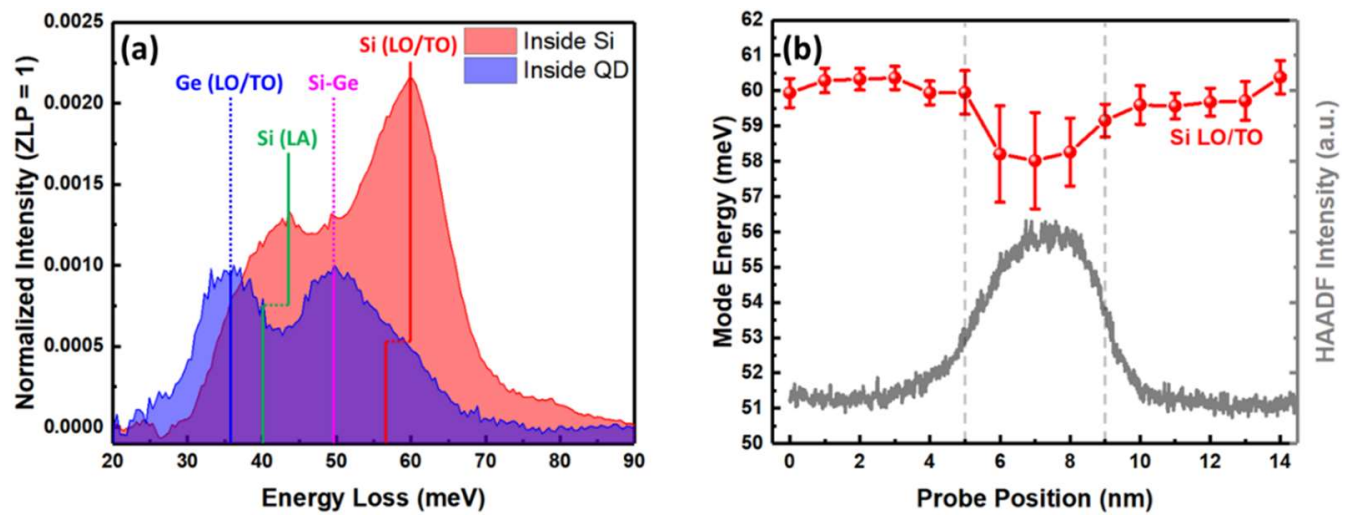

Figure 2. Phonon response of a single Si/Ge QD. (a) Background subtracted phonon signals. The red color denotes the signal in pure $\mathrm{Si}$ while the blue represents the signal when the probe is placed inside the $\mathrm{Si} / \mathrm{Ge} \mathrm{QD}$. (b) A nanometer step line scan across a single QD, along with corresponding HAADF intensity. 